\title{
La innovación tecnológica como repuesta integral para enfrentar el cambio climático
}

\section{The technological innovation as an integral response to face the climate change}

\author{
Delia del Socorro Moreno ${ }^{1}$
}

\section{RESUMEN}

El cambio climático es el desafío ambiental más complejo y de mayor gravedad que actualmente, enfrenta la humanidad, convirtiéndose en una de las tareas agendadas de alta prioridad de los gobiernos miembros de la Comisión Marco de las Naciones Unidas sobre el Cambio Climático (CMNUCC). Estas medidas requieren acciones colectivas para estabilizar los niveles de gases de efecto invernadero (GEI), así como políticas de regulación para limitar las emisiones y fomentar el impulso de acciones de innovación para la mitigación y adaptación al cambio climático. Acertadamente la innovación tecnológica es una aliada estratégica y sumamente necesaria para enfrentar este flagelo que pone en riesgo la vida humana. Por la complejidad de estos procesos, conlleva a tomar en cuenta las políticas de desarrollo de los países y los recursos disponibles para incentivar la innovación tecnológica que materialice la adopción de las mismas basado en las realidades y condiciones de cada territorio.

Palabras clave: innovación tecnológica; cambio climático; adaptación al cambio climático; políticas de desarrollo.

\begin{abstract}
Climate change is the most complex and gravest environmental challenge faced by humanity currently, becoming one of the high priority tasks of the government members of the United Nations Framework Commission on Climate Change (UNFCCC). These actions require collective actions to stabilize greenhouse gas (GHG) levels, as well as regulatory policies to limit the emissions and encourage the promotion of innovated actions for the mitigation and adaptation to climate change. Accurately the technological innovation is a strategic helper and extremely necessary to face this infliction that risk human life. Due to the complexity of these processes, it is necessary to take into account the development policies of the countries and the available resources to encourage to the technological innovation that materializes their acceptance based on the realities and conditions of each territory.
\end{abstract}

Keywords: technological innovation; climate change; adaptation to climate change; development policies.

DOI: https://doi.org/10.5377/multiensayos.v3i6.9693

Recibido: 15 de diciembre de 2017

Aceptado: 03 de enero de 2018

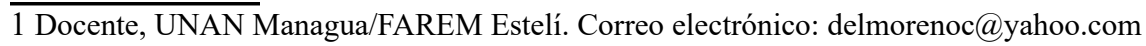




\section{INTRODUCCIÓN}

La adopción de tecnologías para la adaptación al cambio climático implica una combinación de políticas de regulación que definan claramente los límites de las emisiones de gases de efecto invernadero (GEI); así como el establecimiento de incentivos a individuos, gobiernos y empresas para fomentar la innovación y la implantación de tecnologías que permitan la adaptación. Estas políticas deben contar con el respaldo financiero para la difusión del conocimiento a través de la formación y la capacitación para asegurar la adopción tecnológica entre otras medidas.

En este sentido, la innovación tecnológica requiere de recursos para promover la investigación orientada a encontrar las posibles rutas para impulsar las innovaciones de reducción de los GEI. Por lo tanto, estos procesos requieren del incremento presupuestario para responder a los retos del cambio climático en base a las necesidades y compromisos de cada realidad territorial.

En los esfuerzos de reducción de las emisiones necesitaremos tecnologías que aún no se han desarrollado o implementado a una escala significativa, o que todavía no hemos inventado. En muchos casos, también se necesitarán innovaciones en sistemas sociales e institucionales que influyen en la demanda energética y en las emisiones (Rubin, 2015).

Esto implica realizar grandes esfuerzos en la investigación, innovación, difusión y adopción para dar respuesta a los desafíos concretos que implica la innovación tecnológica e idear oportunidades que contribuyan a la mitigación y adaptación del cambio climático, significando un importante desafío a todos los niveles.

\section{DESARROLLO}

\section{Cambio Tecnológico en las emisiones}

En los diversos eventos del clima se ha debatido en cuanto a la génesis del cambio climático, ya que por un lado están los procesos naturales (emisiones de CO2 de los océanos y/o los ciclos de carbono) y por otra parte las actividades antropogénicas (las emisiones de los gases invernaderos (Lugo Marín, 2015, pág. 6). Estos gases de efecto invernadero (GEI), son de origen natural y antropogénico, están conformados por el vapor de agua (H2O), el dióxido de carbono $(\mathrm{CO} 2)$, el dióxido de nitrógeno (NO2), el metano (CH4) y el Ozono (O3). Los gases incorporados a la atmósfera producidos por los seres humanos los clorofluorocarbonos (CFC), hidroflurocarbono (HFC) y hexafluoruro de azufre (SF6).

En el informe especial del IPCC sobre escenarios de emisiones (IPCC, 2000), proyecta un aumento de las emisiones mundiales de GEI de entre $25 \%$ y $90 \%$ (CO2) entre 2000 y 2030 , suponiendo que los combustibles de origen fósil mantengan su posición dominante en el conjunto mundial de fuentes de energía hasta 2030 como mínimo. Otros escenarios más recientes, que no contemplan medidas de mitigación de las emisiones adicionales, arrojan resultados similares. 
A pesar de esto, las emisiones globales de gases de efecto invernadero continúan creciendo y la temperatura media mundial no deja de aumentar. Prueba de ello es que el 2016 fue el año más caliente desde que se tienen datos, y la temperatura media mundial está un grado por encima de los niveles preindustriales. Para alcanzar los objetivos del Acuerdo de París, se deben implantar tecnologías climáticas a una escala mucho mayor y, aquí, la innovación puede jugar un papel clave (ONU, 2017).

Por su parte (OpenMind, 2017), expone que las emisiones de GEI dependen fundamentalmente de los tipos de fuentes de energía y de las tecnologías que se emplean para suministrar los artículos y los servicios que la sociedad necesita. Así, las innovaciones tecnológicas pueden ayudar a reducir las emisiones de GEI de diversas formas (NRC, 2010, pág. 202). Por ejemplo, plantea tres aspectos:

- Las tecnologías nuevas o mejoradas pueden hacer que bienes como los vehículos, la maquinaria y los electrodomésticos usen la energía de un modo más eficiente, lo que reduce su consumo energético y las emisiones de GEI por unidad de producto o servicio útil (como un vehículomilla de viaje o un lumen de luz en el caso de la iluminación).

- Las nuevas tecnologías pueden crear o utilizar vectores de energía y agentes químicos alternativos que emitan menos GEI por unidad de producto o servicio útil (como las fuentes de energía renovables o los nuevos fertilizantes con bajo contenido de nitrógeno).

- Las nuevas tecnologías pueden crear formas alternativas de suministrar bienes y servicios caracterizadas por una menor generación de GEI (ya sea mediante el uso de productos o materiales sustitutivos que emitan menos GEI o a través de cambios de mayor alcance que afecten a todo el sistema, como el reemplazo de los desplazamientos en coche o en avión por las teleconferencias y el teletrabajo).

Esto implica que solo mediante acciones gubernamentales que exijan o hagan viable desde el punto de vista financiero reducir las emisiones de GEI se pueden crear mercados de cierta entidad para los productos y los servicios que posibilitan tales reducciones. Por tanto, las acciones gubernamentales dirigidas a crear o ampliar los mercados de las tecnologías de reducción de las emisiones de GEI son esenciales en el proceso de innovación tecnológica (OpenMind, 2017).

A partir de estos planteamientos es sumamente urgente y necesario un cambio tecnológico para contribuir al alcance del objetivo de estabilizar los niveles de emisiones de gases de efecto invernadero (GEI), para ello habrá que centrar esfuerzos en el reemplazo de las tecnologías actuales que no contribuyen a la disminución de estos gases peligrosos, especialmente las que están basadas en el uso de combustible fósiles (petróleo, gas y carbón).

En muchos casos afirma (Rubin, 2015), este proceso requerirá tecnologías avanzadas que aún no se han desarrollado o no se han adoptado a una escala comercial apreciable o que ni siquiera se han inventado. 
Esto obviamente es una oportunidad para orientar la investigación hacia la innovación de tecnologías de contribuyan de manera importante en productos o servicios para la mitigación y adaptación al cambio climático. Ambas son desafíos importantes que precisan de soluciones basadas en la innovación tecnológica amigable con el medio ambiente.

\section{La innovación tecnológica para la adaptación y la mitigación ante el Cambio Climático}

Según (March, 2008), la mitigación consiste en frenar el calentamiento del planeta reduciendo el nivel de gases de efecto invernadero que se liberan a la atmósfera. Entre las diversas tecnologías de mitigación que ya se comercializan o están a punto de salir al mercado, se encuentran las fuentes de energía renovables, como los biocarburantes, la biomasa y la energía eólica, solar e hidráulica; el material de construcción con bajo nivel de emisiones de carbono y las tecnologías emergentes destinadas a captar el carbono de la atmósfera para almacenarlo de forma segura.

En el caso de la adaptación consiste en paliar las repercusiones previstas o las que ya ha ocasionado el cambio climático, en particular en los países en desarrollo, los países menos adelantados y las islas pequeñas, que son los más afectados. Además de las tecnologías "inmateriales", como la alternancia de cultivos, entre las tecnologías "materiales" de adaptación se encuentran las técnicas de regadío perfeccionadas para combatir las sequías y las obtenciones vegetales resistentes a la sequía o al agua salada (March, 2008).

En ese sentido la Agencia Internacional de energía calcula que al 2020, el 60 por ciento de las emisiones de GEI provendrá de economías en transición y de países en desarrollo y hace énfasis en que estos países tendrán que saltarse una o dos generaciones tecnológicas para evitar la trampa de los combustibles fósiles y pasar directamente a las tecnologías racionales desde el punto de vista medio ambiental.

Sin embargo, para un cambio tecnológico en el nivel que se requiere se necesita influir en un mercado con tecnologías con bajos niveles de carbono cuyo único fin es la reducción de GEI, que son más caras que las opciones actuales.

Las innovaciones tecnológicas pueden favorecer todo este espectro de posibilidades. Un conjunto aún más amplio de innovaciones incluiría, además, sistemas y diseños sociales e institucionales. Por ejemplo, las innovaciones en el planeamiento y el desarrollo urbanístico podrían ayudar a reducir la demanda energética futura (y las emisiones de GEI asociadas a ella) en el transporte y en los edificios residenciales y comerciales. Las innovaciones institucionales podrían proporcionar incentivos para que las compañías de suministro eléctrico y otras empresas inviertan en medidas que reduzcan la demanda energética, en lugar de guiarse por políticas dirigidas a aumentar la venta de energía ( (OpenMind, 2017). 
En este caso es sumamente urgente acciones gubernamentales que impulsen y respalden financieramente la reducción de emisiones de GEI, se podrían crear mercados para los productos y servicios que estén orientados a la reducción en el proceso de innovación tecnológica.

En otro orden, (ICTSD, 2009), manifiesta que los países en desarrollo necesitan acceso tanto a las últimas tecnologías ambientales como a toda la información técnica y comercial para poder entenderlas, usarlas y desarrollarlas. Esto es fundamental no sólo con base en el principio de responsabilidades comunes pero diferenciadas sino como elemento esencial de una transición mundial hacia una estructura económica más sostenible. Sin embargo, la magnitud y rapidez del cambio climático pone en evidencia que los acuerdos multilaterales ambientales no pueden concluir allí la consideración de este tema, se requiere explorar a fondo la posibilidad de guiar y estimular el cambio tecnológico desde la innovación hasta el amplio acceso a las nuevas tecnologías

\section{Las políticas gubernamentales y la innovación ante el Cambio Climático}

En el informe presentado por (IPCC, 2007), se expone que el apoyo gubernamental a través de contribuciones financieras, créditos tributarios, fijación de normas, y creación de mercado es importante para el desarrollo de energías eficientes, la innovación y el despliegue. La transferencia de tecnología a los países en desarrollo depende de las condiciones que la posibiliten y de la financiación.

También afirman que el flujo financiero a los países en desarrollo a través de los proyectos de MDL (Mecanismos de Desarrollo Limpio) tiene el potencial de alcanzar niveles del orden de varios billones de dólares estadounidenses por año, el cual es mayor que el flujo a través del Fondo para el Medio Ambiente Mundial (FMAM), comparable con el flujo de asistencia para el desarrollo orientado a la energía, pero al menos de un orden de magnitud más bajo que el total de los flujos de inversiones extranjeras directas. Los flujos financieros del MDL y del FMAM y la asistencia al desarrollo para transferencia de tecnología han sido, hasta el presente, muy limitados, y se han distribuido geográficamente de forma desigual.

En este sentido (OpenMind, 2017), explica que las políticas gubernamentales desempeñan un papel especialmente importante en el fomento de las innovaciones con las que se intenta responder al problema del cambio climático. De tal manera que para alcanzar las grandes reducciones de las emisiones de GEI se necesita aminorar los riesgos asociados al cambio climático, y para ello se debe contar con políticas orientadas no solo al fomento de la innovación tecnológica, sino también la adopción de las nuevas tecnologías por todos los involucrados en estos procesos de desarrollo. Estas políticas deben contar con apoyo financiero y la formación del capital humano.

Sobre el capital humano (Rubin, 2015), refiere que las emisiones requerirá un número mayor de trabajadores especializados, sobre todo técnicos y científicos, pero también de ciencias sociales. Tanto el sector público como el privado tienen un importante papel que jugar, atrayendo y 
reteniendo a los mayores talentos del mundo para que estudien los desafíos e inventen nuevas formas de mitigar el cambio climático global.

Ala vez se requiere un incremento de apoyo a la innovación que facilite la inversión en las tecnologías que los países requieren para la mitigación y adaptación al cambio climático, lo cual implica la creación de mecanismos ágiles en el acceso a los recursos disponibles para los países en vías de desarrollo.

\section{CONCLUSIONES}

Las tecnologías y su adopción son componentes esenciales para la respuesta integral al cambio climático es un proceso complejo que conlleva la interacción entre las distintas fases que integran la invención, innovación, adopción y difusión. La adopción misma es un proceso que requiere un esfuerzo a largo plazo. Las políticas facilitan los resultados en cada fase de este proceso.

De igual manera, es importante la difusión del conocimiento a través del soporte financiero a la educación y formación encaminadas a la adopción de estas tecnologías, esfuerzos que requieren de políticas coherentes, de apoyo al campo de la investigación y de acceso a recursos para incentivar la innovación en relación al cambio climático.

La innovación sirve como una fuerza positiva para el cambio en el estímulo a la creatividad y el desarrollo de la investigación para fortalecer la mitigación y adaptación al cambio climático, facilitando la generación de políticas que contribuirán a aminorar los riesgos asociados a esta problemática. En este contexto, es sumamente urgente incidir en pedagogía, conciencia y comunicación para interiorizar en la necesidad de innovar para enfrentar el cambio climático.

\section{BIBLIOGRAFIA}

ICTSD. (9 de Marzo de 2009). Tecnología y cambio climático: ¿un nuevo enfoque para el derecho ambiental? Obtenido de Tecnología y cambio climático: ¿un nuevo enfoque para el derecho ambiental?: http://www.ictsd.org/bridges-news/puentes/news/tecnolog\%C3\%ADa-ycambio-clim $\% \mathrm{C} 3 \% \mathrm{~A} 1$ tico-\%C2\%BFun-nuevo-enfoque-para-el-derecho-ambiental

IPCC. (2000). Escenario de Emisiones. España.

IPCC. (2007). Informe del Grupo de Trabajo III - Mitigación del Cambio Climático. Obtenido de E. Políticas, medidas e instrumentos para mitigar el cambio climático: https://www.ipcc. ch/publications_and_data/ar4/wg3/es/spmse.html

Lugo Marín, D. D. (2015). Bases Teóricas, Racionalidad ambiental, Causas y Evidencias y Escenarios del Cambio Climático. España.

March, E. (Febrero de 2008). Revista de la OMPI. Obtenido de El cambio climático - Un desafío tecnológico.: http://www.wipo.int/wipo_magazine/es/2008/01/article_0001.html

NRC. (2010). National Research Council, Limiting the Magnitude of Climate Change, Washington. 
National Research Council, Limiting the Magnitude of Climate Change, Washington,: The National Academies Press.

ONU. (2 de Mayo de 2017). ONU Cambio climático Acción Climática. Obtenido de Innovación tecnológica, clave frente al cambio climático: http://newsroom.unfccc.int/es/accionclimatica/innovaci\%C3\%B3n-tecnologica-clave-frente-al-cambio-climatico/

OpenMind. (5 de Mayo de 2017). Compartiendo conocimiento para un futuro mejor. Obtenido de Innovación y Cambio Climático: https://www.bbvaopenmind.com/articulo/innovacion-ycambio-climatico/?fullscreen $=$ true

Rubin, E. (2015). Innovación, la clave para combatir el cambio climático. CINCO DIAS, 11-12. Obtenido de CINCO DIAS. 